Е. В. Жужома, В. С. Медведев, О типичной диффеотопии грубого диффеоморфизма с растягивающимся аттрактором коразмерности один, Матем. заметки, 2003, том 74, выпуск 3, 478-480

DOI: https://doi.org/10.4213/mzm596

Использование Общероссийского математического портала Math-Net.Ru подразумевает, что вы прочитали и согласны с пользовательским соглашением http://www.mathnet.ru/rus/agreement

Параметры загрузки:

IP : 54.92 .164 .108

26 апреля 2023 г., 02:49:59 


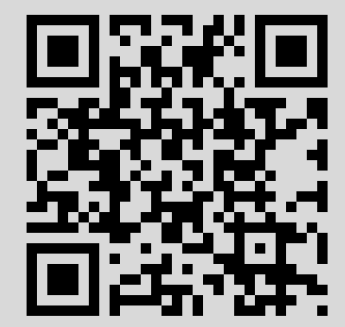




\section{О ТИПИЧНОЙ ДИФФЕОТОПИИ ГРУБОГО ДИФФЕОМОРФИЗМА С РАСТЯГИВАЮЩИМСЯ АТТРАКТОРОМ КОРАЗМЕРНОСТИ ОДИН}

\section{Е.В. Жужома, В.С. Медведев}

Пусть $M^{n}$ - замкнутое $n$-многообразие $(n \geqslant 2)$, и $\operatorname{Diff}^{r}\left(M^{n}\right)$ - пространство $C^{r}$ гладких $(r \geqslant 1)$ диффеоморфизмов многообразия $M^{n}$, наделенное равномерной $C^{r}$-топологией (основные понятия и факты теории динамических систем можно найти в обзорах [1], [2] и [3]). Под $C^{k}$ - дuффеотопией диффеоморфизма $f_{0} \in \operatorname{Diff}^{r}\left(M^{n}\right), 0 \leqslant k \leqslant r$, понимается отображение $f:[0 ; 1] \times M^{n} \rightarrow$ $M^{n}$, которое $C^{k}$-гладко зависит от первого множителя $\mu \in[0 ; 1]$, и для каждого $\mu \in[0 ; 1]$ отображение $f(\mu, \cdot): M^{n} \rightarrow M^{n}$ является диффеоморфизмом из $\operatorname{Diff}^{r}\left(M^{n}\right)$. Иногда диффеотопию называют $C^{k}$ - дугой в пространстве $\operatorname{Diff}^{r}\left(M^{n}\right)$ [4]-[7]. Множество дуг естественным образом наделяется $C^{k}$-топологией. Везде далее считаем $k=1$.

Грубые (или структурно устойчивые) диффеоморфизмы существуют на любом замкнутом многообразии и образуют открытое подпространство в $\operatorname{Diff}^{r}\left(M^{n}\right)$. Поэтому естественно рассмотреть дуги, удовлетворяющие некоторым условиям типичности, между грубыми диффеоморфизмами. В известном списке пятидесяти проблем Дж. Пэлиса и Ч. Пью [7] в проблеме 33 поставлен вопрос о существовании типичной в некотором смысле дуги в пространстве динамических систем между двумя структурно устойчивыми динамическими системами (векторными полями или изотопными диффеоморфизмами). Под типичной дугой обычно понимается устойчивая, умеренно устойчивая или простая дуга. Дадим необходимые определения.

Две дуги (или диффеотопии) назьваются топологически сопряжсенными, если, с точностью до сохраняющего ориентацию гомеоморфизма отрезка $I=[0 ; 1]$, любые диффеоморфизмы этих дуг, соответствуюшие одинаковому параметру, сопряжены и сопрягающий гомеоморфизм непрерывно зависит от параметра $\mu \in[0 ; 1]$. Если сопрягающий гомеоморфизм необязательно зависит от параметра непрерьвно, то такие диффеотопии называются умеренно сопряжсенными. Топологическая сопряженность и умеренная сопряженность являются отношениями эквивалентности и разбивают множество дуг на классы эквивалентности. Внутренности этих классов образуют $y c-$ тойчивые и умеренно устойчивые дуги диффеоморфизмов соответственно (см. [5]). Устойчивая дуга называется простой, если она содержит толко конечное множество бифуркационных точек (т.е. точек, соответствующих негрубым динамическим системам) [4].

Один из замечательных классов грубых диффеоморфизмов составляют диффеоморфизмы Аносова. Непосредственньм развитием диффеоморфизмов Аносова коразмерности один являются грубые А-диффеоморфизмы с базисными множествами коразмерности один (коразмерность один означает, что топологическая размерность базисного множества на единицу меньше размерности объемлющего многообразия). Если к диффеомор физму Аносова $n$-мерного тора $T^{n}$ коразмерости один применить хирургическую операцию, то получим грубый А-диффеоморфизм с нетривиальным базисньм множеством коразмерности один, локально гомеоморфньм произведению гиперплоскости

Работа выполнена при финансовой поддержке Российского фонда фундаментальных исследований, грант № 02-01-000-98. 
коразмерности один на канторово множество. В зависимости от исходного диффеоморфизма Аносова полученное нетривиальное базисное множество $\Omega$ является либо ориентируемым расстягивающимся аттрактором, либо сжимающимся репеллером. В каждой компоненте множества $T^{n}-\Omega$ лежит ровно одна изолированная периодическая точка. Следуя Смейлу [3], будем назьвать полученный А-диффеоморфизм ДА-диффеоморфизмом.

В работе рассматриваются типичные дуги, позволяющие переходить от одного грубого диффеоморфизма с ориентируемьм растягивающимся аттрактором (или сжимающимся репеллером) коразмерности один к изотопному ему грубому диффеоморфизму с ориентируемым растягиваюшимся аттрактором (или сжимающимся репеллером) коразмерности один.

Отметим, что Ньюхаус и Пейкшото [6] доказали, что любые потоки Морса-Смейла на фиксированном замкнутом многообразии соединяются простой дугой. Этот результат был обобщен Ньюхаусом [4] на потоки с так назьваемьми $d$-разделенньми базисными множествами. Что касается диффеоморфизмов, то здесь ситуация не столь ясная. Например, два грубых диффееоморфизма окружности с разньми числами вращения нельзя соединить простым путем в пространстве диффееморфизмов окружности. Матсумото [8] доказал, что на любой ориентируемой замкнутой поверхности рода $\geqslant 1$ существует счетное семейство изотопных тождественному диффеоморфизмов МорсаСмейла, которые попарно не соединяются в пространстве диффеоморфизмов простым путем.

Напомним необходимые для дальнейшего определения. Пусть $f-$ А-диффеоморфизм замкнутого $n$-мерного многообразия $M^{n}$. Известно [3], что множество неблуждающих точек $N W(f)$ представляется в виде конечного объединения попарно непересекающихся замкнутых инвариантных множеств $\Omega_{1}, \ldots, \Omega_{k}$, называемых базисными множсествами, каждое из которых содержит всюду плотную орбиту.

Базисное множество $\Omega$ назьвается аттрактором, если существует окрестность $U$ этого множества такая, что $\cap_{j \geqslant 0} f^{j}(U)=\Omega$. Множество называется репеллером, если оно является аттрактором для $f^{-1}$. Ясно, что многие результаты об аттракторах можно перефразировать применительно к репеллерам, и наоборот. Под размерностью базисного множества мы понимаем топологическую размерность. Аттрактор $\Omega$ назьвается растягивающимся, если размерность $\Omega$ совпадает с размерностью неустойчивого многообразия любой его точки.

Согласно теореме 1 [9], базисное множество $\Omega$ коразмерности один является либо аттрактором, либо репеллером. В этом случае неустойчивое (если $\Omega$ - аттрактор), либо устойчивое (если $\Omega$ репеллер) многообразие любой точки $x \in \Omega$ принадлежит $\Omega$. Согласно теореме 2 [9] растягивающийся аттрактор или сжимающийся репеллер коразмерности один имеет локальную структуру прямого произведения $(n-1)$-мерного евклидова пространства и канторова множества.

Для точки $x \in M^{n}$ обозначим через $W^{u}(x), W^{s}(x)$ неустойчивое и устойчивое многообразия, проходящие через эту точку. Иногда мы будем обозначать эти многообразия через $W^{s(u)}(f, x)$, указьвая явно диффеоморфизм $f$. Каждое $W^{s}(x), W^{u}(x)$ есть образ евклидового пространства относительно гладкой иммерсии (т.е. $W^{s}(x)$ и $W^{u}(x)$ являются имерсированньми подмногообразиями). Обозначим через $W_{R}^{s(u)}(x)$ диск в подмногообразии $W^{s(u)}(x)$ радиуса $R>0$ с центром в точке $x$. Открытые диски $W_{\alpha}^{s}(x), W_{\beta}^{u}(x)$ являются ориентируемьми и нормально ориентируемьми имерсированньми подмногообразиями дополнительной размерности, $\operatorname{dim} W_{\alpha}^{s}(x)+\operatorname{dim} W_{\beta}^{u}(x)=$ $n$, для любых $\alpha>0, \beta>0$. Следовательно, корректно следующее определение ориентируемости (по Гринесу) базисного множества. Будем говорить, что базисное множество $\Omega$ ориентируемо, если для любой точки $x \in \Omega$ и любых фиксированных чисел $\alpha>0, \beta>0$ индекс пересечения $W_{\alpha}^{s}(x) \cap W_{\beta}^{u}(x)$ во всех точках пересечения один и тот же $(+1$, либо -1$)[10],[11]$. В противном случае базисное множество $\Omega$ назьвается неориентируемым. Основной результат содержится в следующей теореме.

Теорема 1. Любой грубый диффеоморфизм $f \in \operatorname{Diff}^{r}\left(M^{n}\right), \quad n \geqslant 4$, с ориентируемым растягивающимся аттрактором коразмерности один соединяется простой дугой с некоторым ДА-диффеоморфизмом.

Используя замечание В) [5, с. 51] о существовании умеренно устойчивой дуги между ДА-диффеоморфизмом и изотопным ему аносовским диффеоморфизмом, можно вывести следующее следствие.

СлЕДСтвИЕ 2. Любъе изотопные друг другу грубъе диффеоморфизмы замкнутого многообразия $M^{n}, \quad n \geqslant 4$, с ориентируемыми растягивающимися аттракторами коразмерности один соединяются умеренно устойчивой дугой. 
Отметим, что для $n=2$ теорема 1 неверна, так как существют грубые диффеоморфизмы с растягивающимися аттракторами коразмерности один на любой замкнутой ориентируемой поверхности $M^{2}$ рода $\geqslant 2$, которые не изотопны никакому ДА-диффеоморфизму. Следствие 2 также, вообще говоря, неверно для $n=2$, поскольку, используя упомянутый результат [8], на любой ориентируемой замкнутой поверхности $M^{2}$ рода $\geqslant 1$ можно построить изотопные друг другу грубые диффеоморфизмы с ориентируемьми растягивающимися аттракторами коразмерности один, которые не соединяются умеренно устойчивыми дугами.

Что касается размерности $n=3$, то в силу существования для этой размерности “диких" одномерных сепаратрис седловых неподвижных точек [12] наше доказательство не проходит (см. ниже лемму 3). Отметим только, что в этой размерности не существует грубых диффеоморфизмов с неориентируемыми растягивающимися аттракторами коразмерности один [13]. Вопрос о существовании таких аттракторов у грубых диффеоморфизмов в размерности $n \geqslant 4$ остается открытым. Основным техническим результатом в доказательстве теоремы 1 является следующая лемма.

ЛЕмма 3. Пусть $\sigma_{0}-$ изолированная седловая периодическая точка диффеоморфизма $f \in \operatorname{Diff}^{r}\left(M^{n}\right)$ такая, что $\operatorname{dim} W^{s}\left(\sigma_{0}\right)=n-1 u \operatorname{dim} W^{u}\left(\sigma_{0}\right)=1$. Предположим, что существует сепаратриса $W_{1}^{u}\left(\sigma_{0}\right) \subset W^{u}\left(\sigma_{0}\right)-\sigma_{0}$ и гиперболический периодический сток $S_{1}$ такой, что $\operatorname{clos} W_{1}^{u}\left(\sigma_{0}\right)=\sigma_{0} \cup W_{1}^{u}\left(\sigma_{0}\right) \cup S_{1}$. Тогда

1) $\operatorname{clos} W_{1}^{u}\left(\sigma_{0}\right)$ есть ручной одномерныи й полиэдр;

2) существует гладко влохенная $(n-1)$-сфера $S^{n-1} \subset W^{s}\left(S_{1}\right)$, ограничивающая $n$-иар, скажем $B^{n}$, такой, что $S_{1} \in \operatorname{int} B^{n}, f^{p}\left(S^{n-1}\right) \subset \operatorname{int} B^{n}$, и пересечение $W_{1}^{u}\left(\sigma_{0}\right) \cap S^{n-1}$ состоит из одной точки, где $p-$ период $S_{1}$;

3) сущ,ствует устойчивая дуга $f_{\mu} \in \operatorname{Diff}^{r}\left(M^{n}\right), 0 \leqslant \mu \leqslant 1, f_{0}=f$, без бифуркационных точек такая, что

a) $N W\left(f_{\mu}\right)=N W(f)$ u $\left.f_{\mu}\right|_{N W\left(f_{\mu}\right)}=\left.f\right|_{N W\left(f_{\mu}\right)}$ для всех $0 \leqslant \mu \leqslant 1$;

б) существует сепаратриса $W_{1}^{u}\left(f_{1}, \sigma_{0}\right) \subset W^{u}\left(f_{1}, \sigma_{0}\right)-\sigma_{0}$ седловой точки $\sigma_{0}$ диффеоморфизма $f_{1}$ такая, что clos $W_{1}^{u}\left(f_{1}, \sigma_{0}\right)=\sigma_{0} \cup W_{1}^{u}\left(f_{1}, \sigma_{0}\right) \cup S_{1}$;

в) дуга clos $W_{1}^{u}\left(f_{1}, \sigma_{0}\right)$ имеет касательную в точке $S_{1}$ (m.e. cenаратриса $W_{1}^{u}\left(f_{1}, \sigma_{0}\right)$ подходит $\kappa S_{1}$ в определенном направлении).

Для доказательства теоремы 1 далее применяются результаты работ [14], [15] о структуре ориентируемых растягивающихся аттракторов коразмерности один грубых диффеоморфизмов.

Авторы благодарят Д. В. Аносова и В.З. Гринеса за полезные обсуждения. Первый автор работал над статьей во время визита в университет г. Рен (Франция), поддержанного CNRS. Он благодарит А. Зорича, И. Итенберга и В. Каймановича за плодотворные дискуссии и тепльй прием.

\section{СПИСОК ЦИТИРОВАННОЙ ЛИТЕРАТУРЫ}

1. Аносов Д. В. // Тр. МИАН СССР. 1985. №169. С. 59-93. 2. Аносов Д. В., Солодов В. В. // Итоги науки и техники. Современные проблемы математики. Фундамент. направления. Динамические системы-9. Т. 66, 1991. С. 12-99. 3. Smale S. // Bull. Amer. Math. Soc. 1967. V. 73. № 1. P. 741-817; // Перевод на рус. язык: УМH. 1970. T. 25. C. 113-185. 4. Newhouse S. // Lecture Notes in Math. 1975. V. 468. P. 209-233. 5. Newhouse S., Palis J., Takens F. // IHES. Publ. Math. 1983. V. 57. P. 5-71. 6. Newhouse S., Peixoto M. M. // Asterisque. 1976. V. 31. P. 15-41. 7. Palis J., Pugh C. // Lecture Notes in Math. 1975. V. 468. P. 345-353. 8. Matsumoto Sh. // Invent. Math. 1979. V. 51. Р. 1-7. 9. Плыкин Р. В. // Матем. сб. 1971. Т. 84. С. 301-312. 10. Гринес В.З. // УМН. 1974. Т. 29. №6(180). С. 163-164. 11. Гринес В.З. // Тр. ММО. 1975. № 32. C. 35-60. 12. Pixton D. // Topology. 1977. V. 16. P. 167-172. 13. Ж Кужома Е. B., Медведев В. С. // Матем. сб. 2002. Т. 193. №6. С. 83-104. 14. Гринес В. З, ЖКужома Е. В. // Докл. РАН. 2000. Т. 374. №6. С. 274-276. 15. Гринес В. З, ЖКужома Е. В. // Изв. РАН. Сер. матем. 2002. Т. 66. № 2. С. 3-66.

(Е. В. Жужома) Нижегородский государственный технический университет, 